\title{
Illness Representations of Cancer among Healthy Residents of Kolkata, India
}

\author{
Lala Tanmoy Das ${ }^{1 *}$, Christina D Wagner ${ }^{2}$, Silvia M Bigatti ${ }^{3}$
}

\begin{abstract}
Cancer illness representations and screening history among residents of Kolkata, India, were investigated along with socio-demographic characteristics in an effort to understand possible motivations for health behavior. A total of 106 participants were recruited from community locations in Kolkata, India and completed surveys including demographics, the illness perception questionnaire-revised (IPQ-R), and previous experience with cancer and screening practices. Participants were $51.5 \%$ college educated, $57 \%$ female, $51.5 \%$ full-time employed with average age of 32.7 years ( $R: 18-60$ years). Descriptive statistics were generated for the subscales of the IPQ-R, cancer-screening practices and cancer experience. Correlation analyses were conducted to investigate associations between cancer representations and socio-demographic variables. Univariate ANOVAs were calculated to determine gender differences in IPQ- $R$ subscales and differences between participants who knew someone diagnosed with cancer versus those who did not. While $76 \%$ of participants knew someone with cancer, only $5 \%$ of the sample engaged in cancer screening. Participants perceived cancer as a serious illness with negative emotional valence. Younger age $(r(100)=-.36, p<0.001)$ and male gender $(F(1,98)=5.22, p=0.01$, $\left.\eta^{2}=0.05\right)$ were associated with better illness coherence. Males also reported greater personal control $(F(1$, 98) $=5.34, p=0.02, \eta^{2}=0.05$ ) were associated with better illness coherence. Low screening rates precluded analyses of the relationship between illness representations and cancer screening. Cancer was viewed as a threatening and uncontrollable disease among this sample of educated, middle class Kolkata residents. This view may act as a barrier to seeking cancer screening. Public awareness campaigns aimed at improving understanding of the causes, symptoms and consequences of cancer might reduce misunderstandings and fear, especially among women and older populations, who report less comprehension of cancer.
\end{abstract}

Keywords: Cancer awareness - illness representations - Kolkata residents - cancer screening - risk - tobacco habits

Asian Pac J Cancer Prev, 16 (2), 845-852

\section{Introduction}

Cancer represents a major cause of morbidity and mortality worldwide and a major public health concern (GLOBOCAN, 2012). While incidence of many cancers in India is lower than that of more developed nations, India's cancer burden is expected to double in the next 20 years from nearly 1 million new cases diagnosed in 2012 to 1.7 million new diagnoses by 2035 . This rise in incidence is predicted from many factors including increased population, increased life expectancy and government efforts to control communicable diseases (Takiar et al., 2011). The most common forms of cancer among Indian males include oral, lung, stomach, colorectal, pharyngeal and esophageal (GLOBOCAN, 2012). Among women, breast cancer became the leading cancer diagnosis in 2009, accounting for over one-fifth of female cancer mortality (Mallath et al., 2014). Cervical cancer also exacts a significant toll on Indian women as the second leading cancer diagnosis (GLOBOCAN, 2012) and the leading cancer-related cause of premature death and disability (Bruni et al., 2014). Cancer statistics in city of Kolkata closely reflect the overall patterns of cancer incidence observed at the national level (Marimuthu et al., 2002). In a recent study by Maiti et al. (2012), investigators found that among men diagnosed with or seeking treatment for cancer in a Kolkata hospital, the most common cancers were tobacco related, such as head and neck, lung, oral, and urinary bladder cancer. Among women in the same hospital, breast cancer was most common (Das et al., 2012; Datta et al., 2012), followed by cancer of the cervix, followed by tobacco related cancers.

A significant portion of cancers diagnosed in India and West Bengal appear to be lifestyle dependent. For example, estimates indicate tobacco is responsible for $30-50 \%$ of cancer diagnoses among Indian men and 10-15\% of cancer diagnoses among Indian women (Roychowdhury et al., 2005; NCRP, 2008). Similarly, risk factors of cervical cancer encompass behaviors associated with lifestyle such as poor sexual hygiene, multiparity, and age of first 
birth (Yasmeen et al., 2010; Raychaudhuri and Mandal, 2012). Mortality rates are also high at $68 \%$ of annual incidence owing to delayed diagnosis and suboptimal care (Mallath et al., 2014). Preventative measures can reduce these high mortality rates and projected growth in cancer incidence. These efforts may include raising awareness, making lifestyle modifications, routinely screening for cancer, and changing other aspects of healthcare seeking behavior (Gupta, 2010).

Cancer awareness and knowledge among Indians has been reported as low. In a pilot survey conducted among 900 residents of West Bengal, only 35\% reported they knew cancer symptoms and only $44.67 \%$ were aware of major risk factors (such as tobacco) (Ray and Mandal, 2004). In an investigation of breast cancer awareness by Somdatta and Baridalyne (2008), 88\% of the 333 women surveyed in a resettlement colony in Delhi were aware of cancer as a disease; however, only $56 \%$ were aware of breast cancer as a disease. Among the women aware of breast cancer, $51 \%$ reported at least one symptom of breast cancer and only $35 \%$ mentioned any risk factors. In a 2010 survey aimed at assessing general awareness of cervical cancer among graduate and post-graduate female students in Kolkata, Saha et al. (2010) found that only $20 \%$ of respondents knew that cervical cancer is one of the most prevalent types of cancer in Kolkata and West Bengal, and that they had very little knowledge of the associated risk factors. Additionally, only $11 \%$ of the respondents had ever heard of cervical cancer screening methods such as the Pap smear. In addition to urban, community settings, awareness has also been reported as low among women attending specialized medical clinics. In a survey of 299 women attending an Obstetrics and Gynecology clinic, $57 \%$ of women reported no knowledge of cervical cancer and $89 \%$ reported no knowledge about Pap tests (Roy and Tang, 2008).

Considering these low levels of awareness and limited resources, it comes as no surprise that cancer screening rates in India may be low. Despite mounting evidence that routine cancer screening may significantly reduce some cancer risk (ACS , 2014), in India, public health efforts to encourage cancer screening among lay populations have not been very successful. In a study conducted by Ray and Mandal. (2004) in Kolkata, India, only $8 \%$ of participants agreed to having ever been faced with, seen or participated in any cancer screening or awareness programs. In another study conducted in Kolkata by Roy and Tang. (2008), researchers found that only $10 \%$ of the female participants had received a Pap test at least once, but only two of these tests were performed for screening. Factors that hinder screening efforts in India include absence of screening facilities and equipment, poor literacy, and socio-cultural barriers. Dabash et al. (2005) found that lack of confidentiality and privacy during screening, cultural norms encouraging modesty, and insufficient importance given to women's health issues were significant barriers to cervical cancer screening. They also found that having a positive confirmatory diagnosis of cervical cancer was associated with stigma due to questions of high parity and sexual promiscuity, which prevented women from screening (Satija, 2012). In the future, more directed education programs, awareness campaigns, and government and NGO initiatives to model and implement screening guidelines could, perhaps, contribute to more positive outcomes in screening activity (Raychaudhuri and Mandal, 2012). Past research has identified several factors affecting people's interest to be proactive about preventative care. One of the most factors is each individual's perceptions and understandings of illness (Moser et al., 2013).

The most common and empirically supported theoretical framework used to study illness perceptions is the Self-Regulation Model (Leventhal et al., 1984). This model proposes that people have certain representations of illness (or illness representations) that determine their appraisals of an illness situation that subsequently impact their health behaviors. The model is conceptualized as a parallel processing framework where one arm is dedicated to cognitive processing of an internal or external stimulus and the other is dedicated to processing emotional aspects of the same stimulus. The model describes four dimensions of illness representations. First, the identity dimension refers to the symptoms, labels and other markers that an individual views as being representative of the illness. Second, the causes dimension refers to an individual's perceptions about the etiology and likely causes of illness. The third dimension is timeline, or a person's perceptions of the likely duration of the illness and his or her expectations about the course of the health problem. This scale has been further divided into the components of acute/chronic timeline and cyclical timeline. Acute/chronic refers to whether individuals perceive an illness to be long-term (chronic) or shortterm (acute) while cyclical timeline refers to individuals' perceptions regarding the stability or changeability of an illness (Moss-Morris et al., 2002). The fourth dimension is consequences, conceptualized as an individual's beliefs about the severity of illness and its impact on the physical, emotional, psychological and socioeconomic functioning of the person. A fifth dimension, cure/control, was added later by Lau and Hartman. (1983) to investigate the extent to which individuals believe that their illness is curable and the amount of control they have over their condition. Recent modifications in measurement of illness representations have led to dividing the cure/ control dimension into separate components of personal control and treatment control and adding a dimension called emotional representations to assess the emotional responses generated by illness.

Laypeople 's perceptions of cancer often stem from social constructions of the disease in lay literature and social media (Anagnostopoulos and Spanea, 2005; Del Castillo et al., 2011). For example, a study by Lemal and Van den Bulck. (2011) found that women who had been regularly exposed to cervical cancer messages on the television were three times more likely to perceive a moderate risk and seven times more likely to perceive a large risk of being diagnosed with cervical cancer. Similarly, De Jesus. (2013) found that the quantity of media-based health information is positively associated with health decision-making and medical advice-seeking behavior above and beyond the influence of health literacy 
and language proficiency. The illness representations that individuals hold are important to study, because they affect people's health and healthcare seeking behaviors that can eventually contribute to disease diagnoses and illness outcomes (Rutten et al., 2009). Considering the diversity of folk beliefs and access to biomedical information across the globe, it is important to identify illness representations across cultures to ensure accurate description of the reasons motivating health behavior in various populations.

The study of illness representations among a healthy population is beneficial for many reasons. First, understanding healthy people's illness representations reveals how a particular illness is perceived prior to a personal exposure to a disease, and this understanding can inform future preventative and therapeutic interventions (Karademas et al., 2010). Second, the information can be shared among community members and may supplement existing knowledge. Third, it can also be used to dispel misperceptions through awareness campaigns, encourage healthcare providers to address common misunderstandings and also derive interventions to modify illness representations in both patients and nonpatients (Hagger and Orbell, 2003; Kaptein et al., 2007). Relatively few studies have been conducted in India, nationally and at a state-level, examining lay people's illness representations. This is a serious concern due to the implications of rising cancer rates (Takiar et al., 2011) as well as more far-reaching consequences, such as national healthcare costs. Our search revealed one study that assessed four of Leventhal's illness representations (identity, timeline, consequences, cure/control) among 800 women in Allahabad, India (Grunfeld and Kohli, 2010). Respondents indicated relatively neutral beliefs about the timeframe and consequences associated with cancer and slightly positive beliefs about controllability of breast cancer. Significant differences emerged in illness representations of women residing in rural versus urban settings such that rural women were less likely to identify breast cancer symptoms and reported more negative beliefs about the consequences of breast cancer, its controllability and longer duration of disease. These differences based on residential setting highlight the importance of investigating socio-demographic correlates of illness representations to present a more nuanced picture, particularly in a population as sociodemographically diverse as India.

Studies examining the association of sociodemographic variables such as age, gender, and education level to illness representations report mixed results. Some report that the variables make a significant contribution to illness perceptions (Robb et al., 2004; Lehto, 2007) while other studies among various ethnic communities show no impact (Lau-Walker, 2004). Few studies in Kolkata and the rest of India have investigated and reported the impact of socio-demographic variables on illness representations. Knowing this information can help in formulating targeted health awareness campaigns, screening promotions and therapeutic interventions.

Another variable that has been associated with illness representations is having personally suffered from the disease or having an ill relative (Del Castillo et al.,
2011). Past literature has shown that personal experience with cancer, caring for ill relatives, and witnessing the prognoses of cancer in the family contributes to more negative perceptions of cancer, increases risk-assessment of self, and affects how individuals cope with illness (Lykins et al., 2008). A study conducted by Del Castillo et al. (2011) in a Spanish population showed that people with a relative diagnosed with cancer, compared to those without one, reported significantly more symptoms of cancer (identity illness representations) and more negative emotional reactions to cancer (emotional representation). Illness representations variables such as these continue to be largely under-studied and under-reported in an Indian population.

Information regarding perceptions of cancer among healthy Bengali residents is relatively scarce. The present study was conducted in Kolkata, West Bengal, which is estimated to be the seventh largest city in India (KMC, 2014), with a population of 4,496,694 (Census, 2011). The general population of the city is both religiously and ethnically diverse and educated, with a literacy rate of $81.31 \%$ that makes for a good location to study Indian illness representations.

Thus, the present study was conducted to characterize illness representations of cancer among a healthy cohort of Indians residing in Kolkata, India. This study had four aims, which were exploratory and descriptive in nature. Our first aim was to determine participants' overall cancer experiences and screening practices. Our second aim was to describe their cancer illness representations. Our third aim was to investigate the influence of sociodemographic factors such as gender, age, and education level on illness representations and screening practices. Our final aim was to compare illness representations of participants who knew someone with cancer versus those who did not with the expectation that knowing someone with cancer would influence cancer illness representations and screening practices.

\section{Materials and Methods}

\section{Participants}

In total, 106 participants enrolled in the study with $57 \%$ female and $43 \%$ male. Nearly all participants $(96 \%)$ were born in India. Average age was 32.74 years $(\mathrm{SD}=130.04)$ with a range of 18 to 60 years. Education ranged from 2 to 23 years, with the average completed grade of 13.78 years $(\mathrm{SD}=2.74)$ and $7.8 \%$ of participants reporting less than 12 years of education, $73.5 \%$ reporting $12-15$ years of education, and $18.6 \%$ reporting 16 years and above. Approximately half of the sample $(51.5 \%)$ reported full time employment, with $8.9 \%$ reporting part-time employment, $8.9 \%$ unemployed, $1 \%$ retired, $6.9 \%$ full time homemakers, and $22.8 \%$ students. Our sample was limited in socio-economic and religious diversity with $83.5 \%$ of participants classifying themselves as middle class and $89.7 \%$ reporting Hindu religious affiliation.

\section{Measures}

Demographic questionnaire. This questionnaire was developed by the research team and asked participants to 
report employment status, social class, years of education, religious and ethnic affiliations, birth country, age and gender.

Illness perception questionnaire-revised (Moss-Morris et al., 2002). This survey assesses the five component dimensions (identity, consequences, timeline, control/ cure, and causes) of illness representations proposed by Leventhal's self-regulatory model in addition to illness coherence and emotional representations. Directions were modified to allow healthy participants to indicate their personal views of cancer. For example, each item referencing "my illness" was replaced by "cancer". Additionally, three common misconceptions regarding breast cancer causes were added to the causes scale, including being hit in the breast, fondling/playing with breast, and injury to breast.

All scales, except for identity, ask participants to indicate their level of agreement with a statement on a 5-point scale ranging from strongly disagree to strongly agree. High scores on timeline (acute/chronic and cyclical), consequences, and emotional representations indicate more strongly held negative beliefs about cancer, indicate it is a chronic disease with a cyclical nature, and are associated with negative outcomes and psychological distress. More positive beliefs about controllability of cancer and understanding of cancer are indicated by high scores on the personal control, treatment control and coherence subscales. High scores on the identity and causes subscales indicate stronger endorsement of each item as a symptom or etiological factor of cancer.

Good reliability of subscales has been reported for the IPQ-R, with Cronbach's alphas ranging from 0.79 to 0.89 (Moss-Morris et al., 2002). Tables 1 and 2 provide descriptive statistics and reliability estimates of each scale in the present study. Reliability was adequate for most scales and ranged from Cronbach's alphas of 0.59 (timeline acute/chronic) to 0.83 (causes). While these reliability estimates are somewhat lower than the initial psychometric study of the IPQ-R, they are consistent with other studies conducted among populations outside of the United States (Del Castillo et al., 2011). Several additional studies establish the psychometric properties of the IPQ-R in cancer (Figueiras and Alves, 2007; Del Castillo et al., 2011).

Previous experience with cancer questionnaire. This questionnaire was also developed by the research team and asked participants to indicate whether they had been diagnosed with cancer, knew someone diagnosed with cancer, and engaged in cancer screening. If they answered yes to any questions, space was provided where they could elaborate on the type of cancer they or someone they knew was diagnosed with, the relationship of the person they knew with cancer, and the outcome for the cancer patient.

\section{Procedure}

The university institutional review board approved the present study. Participants were recruited in Kolkata, India from several community locations including railway stations, outside of college premises, places of worship, shopping malls, recreation facilities and food courts. Potential participants were approached by the researcher who described the purpose of the study and asked if they were interested in taking part. If they agreed, they signed a consent form. Participants were then either given a paper copy of the questionnaires to complete on the spot or the researcher went through the questionnaires orally with the respondent and filled in the answers. If participants were not familiar with particular clinical terminologies used in the questionnaires, the researcher provided a brief description. All participants were asked the same questions, regardless of whether the questionnaires were administered orally or in writing.

\section{Statistical analyses}

All data were entered and analyzed using SPSS. Three participants failed to complete over half of the IPQ-R items and were therefore dropped from analyses. Mean substitution was used in cases where participants missed one or two items on an IPQ-R scale. In order to address the descriptive aims of the study outlined as Aims 1 and 2, descriptive statistics were calculated for cancer screening practices, cancer experiences and subscales of the IPQ-R.Aim 3, to evaluate relationships between sociodemographic variables with illness representations and screening practices, was tested with correlation analyses when socio-demographic variables were measured on an interval scale (such as age and education level). Univariate analyses of variance tests (ANOVAs) were conducted when socio-demographic variables were measured on a nominal scale (including gender). Aim 4, comparing participants with previous cancer experience to those without on illness representations and cancer screening was tested with a series of ANOVAs.

\section{Results}

\section{Experience with cancer and cancer screening}

Our first aim was to describe the cancer experiences and cancer screening practices among sample participants. In this sample, only two participants reported a personal cancer diagnosis; one had throat cancer and the other liver cancer. While personal history of cancer diagnosis was limited, most participants $(76 \%)$ reported knowing someone with cancer. Among these, 23\% reported knowing an immediate relative (father, mother, brother, sister, son/daughter) and $63 \%$ reported knowing an extended relative (uncle, aunt, brother/sister-in-law, grandmother, grandfather) who had been diagnosed with cancer. The remaining $14 \%$ of participants knew friends who had received cancer diagnoses.

\section{Cancer illness representations}

Our second aim was to describe cancer illness representations. Cancer identity was characterized by calculating participant agreement with each health symptom listed on the IPQ-R identity scale. Half or more participants endorsed each symptom except breathlessness, nausea, sore eyes, wheeziness, and stiff joints. Loss of strength, pain, and weight loss were the most frequently endorsed symptoms, each with greater than $75 \%$ agreement. See Table 1 for percentage agreement with each symptom measuring cancer identity. 
Table 1 also shows participant responses to 23 possible causes of cancer. Behavioral and environmental risk factors were endorsed highly with participants rating tobacco use, alcohol consumption and environmental pollution as the strongest causes of cancer. The least endorsed causes included personality, family problems and fondling or playing with the breast. Interestingly, a noteworthy minority of participants (12\%) endorsed cancer as a punishment from god and nearly one third (28\%) agreed that cancer was caused by immoral/sinful behavior.

Descriptive statistics were calculated for each IPQ-R subscale. Table 2 shows possible score ranges and reliability estimates for each scale as well as the range of scores and means reported by this sample. Evaluation of means shows that participants endorsed cancer as a serious illness $(M=18.29$ of possible 24 on the consequence scale) with negative emotional connotations ( $M=15.41$ of possible 24 on the emotional representations scale).

\section{Influence of demographics on cancer illness representations and screening}

Our third aim investigated correlates of cancer screening practices and cancer illness representations among participants, as well as identified meaningful demographic differences in cancer representations and screening. We limited our tests to those demographic variables that exhibited variability, which included education level, age and gender. To assess the association between cancer illness representations with age and education level, we conducted correlation analyses. No significant correlations were found between education and IPQ-R scales. A negative correlation was found between illness coherence and age $(\mathrm{r}(100)=-.36, \mathrm{p}<0.001)$, such that younger age was associated with better self-reported understanding of cancer. Univariate analyses of variance (ANOVAs) were conducted to assess gender differences in the IPQ-R scales. Significant gender differences were found for personal control $\left(\mathrm{F}(1,98)=5.34, \mathrm{p}=0.02, \eta^{2}=0.05\right)$ and illness coherence $\left(\mathrm{F}(1,98)=5.22, \mathrm{p}=0.01, \eta^{2}=0.05\right)$. Examination of the means revealed that males felt more personal control or self-efficacy $\left(\mathrm{M}_{\text {control }}=13.69, \mathrm{SD}=4.51\right)$ over cancer and better comprehension $\left(\mathrm{M}_{\text {coherence }}=11.32\right.$, $\mathrm{SD}=2.73)$ of cancer than females $\left(\mathrm{M}_{\text {control }}=11.90, \mathrm{SD}=3.24\right.$; $\mathrm{M}_{\text {coherence }}=9.83, \mathrm{SD}=3.57$ ).

While the low rate of screening practices in this sample precludes inferential statistical analysis, some focused descriptive analyses were performed to better describe the behavior of subsets of this sample. The American cancer society (2014) recommends cancer screening for women at age 40 , with the exception that women should screen for cervical cancer every 3 years after beginning having vaginal intercourse. Screening is recommended for men at age 50 with colonoscopy and PSA. We examined rates of screening behavior among women aged 40 years and older and men 50 years and older. Our sample included 40 men, 5 of which were 50 years or older. Two men $(4.7 \%)$ reported cancer screening; one man was younger than 50 years and one was older. Fifty-seven females responded to items assessing cancer screening and 7 (12.3\%) of these women reported having a cancer screening, which
Table 1. Cancer Symptoms and Causes

\begin{tabular}{|c|c|c|}
\hline Scales & Agr & reemen \\
\hline Identity/Cancer- & Loss of Strength & $84 \%$ \\
\hline Symptoms ${ }^{\mathrm{a}}$ & Pain & $81 \%$ \\
\hline & Weight Loss & $77 \%$ \\
\hline & Bone Pain & $62 \%$ \\
\hline & Sleep Difficulties & $58 \%$ \\
\hline & Dizziness & $56 \%$ \\
\hline & Fatigue & $56 \%$ \\
\hline & Headaches & $56 \%$ \\
\hline & No Symptoms & $54 \%$ \\
\hline & Sore Throat & $53 \%$ \\
\hline & Stomach Upset & $50 \%$ \\
\hline & Breathlessness & $47 \%$ \\
\hline & Nausea & $45 \%$ \\
\hline & Wheeziness & $41 \%$ \\
\hline & Sore Eyes & $34 \%$ \\
\hline & Stiff Joints & $25 \%$ \\
\hline Causes $^{\mathrm{b}}, \alpha=.83$ & Smoking & $87 \%$ \\
\hline & Pollution & $73 \%$ \\
\hline & Alcohol & $70 \%$ \\
\hline & Poor Medical Care & $55 \%$ \\
\hline & Diet/Eating Habits & $53 \%$ \\
\hline & Hereditary & $48 \%$ \\
\hline & Injury to Breast & $40 \%$ \\
\hline & Altered Immunity & $38 \%$ \\
\hline & Germ/Virus & $38 \%$ \\
\hline & Accident/Injury & $33 \%$ \\
\hline & Chance/Bad Luck & $32 \%$ \\
\hline & My Own Behavior & $30 \%$ \\
\hline & Stress/Worry & $28 \%$ \\
\hline & Immoral/Sinful Behavior & $28 \%$ \\
\hline & Aging & $25 \%$ \\
\hline & Mental Attitude (negative thinking) & $15 \%$ \\
\hline & Punishment from God & $12 \%$ \\
\hline & Emotional State (anxious, down) & $12 \%$ \\
\hline & Overwork & $10 \%$ \\
\hline & Fondling Breast & $9 \%$ \\
\hline & Family problems & $8 \%$ \\
\hline & My Personality & $5 \%$ \\
\hline
\end{tabular}

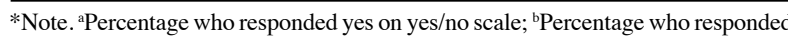
Strongly Agree or Agree on 5-point scale

Table 2. Descriptive Statistics for IPQ-R Scales

\begin{tabular}{lcccc}
\hline Scale & $\begin{array}{c}\text { Possible } \\
\text { Range }\end{array}$ & $\mathrm{R}$ & $\alpha$ & $\mathrm{M}(\mathrm{SD})$ \\
\hline Timeline (acute/chronic) & $0-24$ & $8-22$ & 0.59 & $15.1(3.19)$ \\
Consequence & $0-24$ & $6-24$ & 0.69 & $18.3(3.59)$ \\
Personal Control & $0-24$ & $2-24$ & 0.71 & $12.7(3.87)$ \\
Treatment Control & $0-20$ & $1-20$ & 0.76 & $12.7(3.46)$ \\
Coherence & $0-20$ & $2-18$ & 0.71 & $10.4(3.28)$ \\
Timeline Cyclical & $0-16$ & $0-15$ & 0.64 & $9.29(2.61)$ \\
Emotional Representation & $0-24$ & $7-24$ & 0.77 & $15.4(4.13)$ \\
\hline
\end{tabular}

included three Pap tests, one mammogram, one x-ray, one colonscopy, and one biopsy. Twenty-four of these women were age 40 or older, of which three $(12.5 \%)$ had screened; two reported Pap test and one a biopsy.

\section{Influence of cancer experience on cancer illness representations and screening}

Our fourth aim investigated whether experience with cancer influenced cancer representations or screening. One measurement of cancer experience was whether or not 
participants knew someone who was treated or currently under treatment for cancer. The majority of participants (75.7\%) knew someone diagnosed with cancer. ANOVAs found no significant differences between participants who knew someone diagnosed with cancer and those who did not on IPQ-R subscales. We also examined screening behavior among respondents who reported having an immediate/extended family member with cancer while attributing "heredity" as a cancer cause and found that $97 \%$ of the participants $(n=37)$ who fit into this category did not screen for cancer at all.

\section{Discussion}

We sought to examine illness representations of cancer among a healthy sample of individuals in Kolkata, India. Specifically, our goal was to describe illness representations of cancer among a healthy sample, and examine their relationships with cancer experience, screening practices, age, gender, and education level. Given the very low rates of reported cancer screening among Bengalis and other Indians, where cancer mortality is high and rates of cancer are predicted to rise, it is imperative to determine factors that may guide intervention programs. Illness representations affect health seeking behaviors (Rutten et al., 2009) and are culturally bound (Dein, 2004) and therefore were the focus of the present study.

Although most research on illness representations focus on individuals with a certain illness, the schemas or cognitive representations individuals hold exist before illness happens. For those who are ill, the representations they held before diagnosis will impact their reactions to the diagnosis and behaviors thereafter (Weinman and Petrie, 1997). For those who are healthy, the representations will determine any preventative behaviors they adopt (Figueiras and Alves, 2007). It is therefore important to understand these representations when developing prevention or screening programs.

In spite of the lower incidence of cancer in India, most of our participants knew someone with cancer; in $23 \%$ of these cases, the person with cancer was an immediate relative. This familiarity with the disease may explain why most of the participants endorsed common cancer symptoms, such as pain, weight loss, and fatigue, and well known cancer causes, such as smoking, pollution, and alcohol. On the other hand, common causes such as aging and one's own behavior were endorsed by less than a third of participants. Given that the most prevalent cancers in Kolkata are significantly related to lifestyle (Maiti et al., 2012), the low endorsement of own behavior as an explanatory factor suggests the need for interventions aimed at educating the public; possibly using a stages of change model approach that targets individuals in the contemplation stage of awareness and increases their risk appraisal and understanding of the disease (Sheeran et al., 2014).

This recommendation for approaching preventative efforts is further supported by participants' subscale scores. Score ranges on the IPQ-R subscales were highest in consequence, emotional representation and timeline (acute/chronic) and lowest in coherence, timeline cyclical, and control. These findings suggest that participants felt the disease is highly impactful and distressing, and that the patient has little control over its outcome. In addition, women scored lower on personal control over and comprehension of cancer than men. These findings further point to the need for education and may relate to the low rates of screening and also indicate poor access to screening services. Our findings match those of others who have sought to understand screening behaviors. Basu et al. (2006) interviewed women who were noncompliant with screening programs in Kolkata, India, and found fear of cancer detection and not wanting to test for a disease for which they had no symptoms as the main causes. Although these authors did not specifically use the self-regulation model in their study, their findings fit well with the construct of illness representations, specifically in control, consequences, and identity. Their non-compliant participants were also lower in literacy than the compliant group. In an epidemiological study, Mallath et al. (2014) found higher incidence and death among the lower socioeconomic status population. These are the individuals who both have lower literacy/ education and therefore understanding of the disease, and also less access to quality care. These factors lead them to seek treatment only when the disease is in the advanced stages. Although in our sample we found some variability in terms of education, education did not relate to illness representation scores. Therefore, our findings suggest that even among those with knowledge and access, cancer is viewed as a threatening and uncontrollable disease.

Clearly there is evidence from our findings, supported by others, that the illness representations of cancer among Kolkata residents should be modified through public health interventions aimed at improving understanding of causes, symptoms and consequences of the disease. Since incidence of breast cancer in Kolkata is on the rise, general education of the disease along with efforts, such as those by Rao et al. (2005) to teach women breast selfcare, will help the lower socioeconomic status women prevent more serious outcomes of cancer in spite of having limited access to screening and care. As demonstrated by the findings of Basu et al. (2006) discussed above, education about the benefits of early detection must be a first step to motivate individuals to seek out community screening programs.

There are limitations to this study that impact interpretation of results. Although higher than in other studies (Ray and Mandal, 2004), cancer screening rates in our sample were low, with only $5 \%$ of all participants screening for any type of cancer, $12 \%$ among those 40 years of age and older. The low rates of screening precluded any examination of illness representations as a predictor of screening practices in this sample. This is unfortunate, as one of the strengths of the construct is its ability to predict behavior. A larger sample size, or a larger study targeting specifically those who screen and those who do not, will yield information on their representations and confirm, or not, the usefulness of the model among Indians. Although recruitment was done at various locations to guarantee a demographically diverse 
population, most of the sample reported being 'middle class' in spite of the distribution of educational attainment. According to the national council for applied economic research, the middle class in India represents only $13.1 \%$ of the population (Shukla, 2010), which suggests our sample was not representative of the Indian population in terms of class, or perception of class, and therefore not generalizable to other groups. Our sample was also mostly under 40 years of age. Although this was a problem in that one of our interests was screening, and screening usually begins after age 40, it was also a benefit. Given our conclusions that education about risk is key to improve preventative efforts, our mostly young population gives us a good idea of what to target, at an age range that should be targeted by public health programs aimed at changing the future trends of cancer.

In spite of these limitations, the study reported here provides valuable information for public health and health care professionals who aim to better understand and reduce the cancer burden for the Indian population. These efforts might curtail the projected growth of cancer incidence and reduce the prevalence and impact of the disease.

\section{Acknowledgements}

The authors are grateful to Eric J. Kutscher of Weill Cornell Medical College and Brandy K. Richmond of DePauw University for their guidance and editing of the manuscript.

\section{References}

ACS (American cancer society) (2014). American cancer society guidelines for the early detection of cancer. Accessed July 1. http://www.cancer.org/healthy/findcancerearly/ cancerscreeningguidelines/american-cancer-societyguidelines-for-the-early-detection-of-cancer

Anagnostopoulos F, Spanea E (2005). Assessing illness representations of breast cancer: a comparison of patients with healthy and benign controls. J Psychosom Res, 58, 327-34.

Basu P, Sarkar S, Mukherjee S, et al (2006). Women's perceptions and social barriers determine compliance to cervical screening: results from a population based study in India. Cancer Detect Prev, 30, 369-74.

Bruni L, Barrioneuvo-Rosas L, Serrano B, et al (2014). Human papillomavirus and related diseases in India. Summary report 2014-08-22. ICO information center on HPV and cancer (HPV Information Centre). Accessed on 11/08/14.

Census Organization of India (2011). Kolkata district: census 2011 data. Accessed July 6. http://www.census2011.co.in/ census/district/16-kolkata.html.

Dabash R, Vajpayee J, Jacob M, et al (2005). A strategic assessment of cervical cancer prevention and treatment services in 3 districts of Uttar Pradesh, India. Reprod Health, 2, 11 .

Das S, Sen S, Mukherjee A, Chakraborty D, Mondal PK (2012). Risk factors of breast cancer among women in eastern India: a tertiary hospital based case control study. Asian Pac J Cancer Prev, 13, 4979-81.

Datta K, Choudhuri M, Guha S, Biswas J (2012). Breast cancer scenario in a regional cancer centre in Eastern India over eight years-still a major public health problem. Asian Pac J Cancer Prev, 13, 809-13.
De Jesus M (2013). The impact of mass media health communication on health decision-making and medical advice-seeking behavior of US Hispanic population. Health Commun, 28, 525-9.

Dein S (2004). Explanatory models of and attitudes towards cancer in different cultures. Lancet Oncol, 5, 119-24.

Del Castillo A, Godoy-Izquierdo D, Vazquez ML, Godoy JF (2011). Illness beliefs about cancer among healthy adults who have and have not lived with cancer patients. Int $J$ Behav Med, 18, 342-51.

Figueiras MJ, Alves NC (2007). Lay perceptions of serious illnesses: an adapted version of the revised illness perception questionnaire (IPQ-R) for healthy people. Psychol Health, 22, 143-58.

GLOBOCAN (2012): Estimated cancer incidence, mortality and prevalence worldwide in 2012. World Health Organization (WHO). http://globocan.iarc.fr/Pages/fact_sheets_ population.aspx . Accessed on 12/05/2014.

Grunfeld EA, Kohli N (2010). Beliefs about breast cancer and help-seeking intentions for the disease among women in India. Women Health, 50, 327-41.

Gupta VB (2010). Impact of culture on healthcare seeking behavior of Asian Indians. J Cult Divers, 17,13-9.

Hagger MS, Orbell S (2003). A meta-analytic review of the common-sense model of illness representations. Psychol Health, 18, 141-84.

Kaptein AA, Scharloo M, Helder DI, et al (2007). Quality of life in couples living with huntington's disease: the role of patients' and partners' illness perceptions. Qual Life Res, 16, 793-801.

Karademas EC, Zarogiannos A, Karamvakalis N (2010). Cardiac patient-spouse dissimilarities in illness perception: associations with patient self-rated health and coping strategies. Psychol Health, 25, 451-63.

KMC (Kolkata Municipal Corporation). (2014). Basic statistics of Kolkata. Accessed July 7. https://www.kmcgov.in/ KMCPortal/jsp/KolkataStatistics.jsp.

Lau RR, Hartman KA (1983). Common sense representations of common illnesses. Health Psychol, 8, 167-85.

Lau-Walker M (2004). Relationship between illness representation and self-efficacy. $J$ Adv Nurs, 48, 216-25.

Lehto RH (2007). Causal attributions in individuals with suspected lung cancer: relationships to illness coherence and emotional responses. J Am Psychiatr Nurses Assoc, 13, 109-15.

Lemal M, Van den Bulck J (2011). Television news coverage about cervical cancer: impact on female viewers' vulnerability perceptions and fear. Eur J Public Health, 21, 381-6.

Leventhal H, Nerenz DR, Steele DJ (1984). Illness representations and coping with health threats. In 'Handbook of Psychology and Health', Eds Baum A, Taylor SE, and Singer JE, Hillsdale (NJ): Erlbaum, 219-52.

Lykins ELB, Graue LO, Brechting EH, et al (2008). Beliefs about cancer causation and prevention as a function of personal and family history of cancer: a national, population-based study. Psychooncology, 17, 967-74.

Maiti PK, Jana U, Ray A, et al (2012). Patterns of cancer occurrence in different regions of West Bengal-a hospital based study. J Indian Med Assoc, 110, 445-8.

Mallath MK, Taylor DG, Badwe RA, et al (2014). The growing burden of cancer in India: epidemiology and social context. Lancet Oncol, 15, 205-12.

Marimuthu P, Chakraborty S, Agarwal SC, Monoharan N, Chatterjee M (2002). Trends of cancer prevalence in some districts of West Bengal. Asian Pac J Cancer Prev, 3, 239-42.

Moser RP, Arndt J, Han PK, Waters EA, et al (2013). Perceptions of cancer as a death sentence: prevalence and consequences. 
J Health Psychol, 19, 1518-24.

Moss-Morris R, Weinman J, Petrie KJ, et al (2002). The revised illness perception questionnaire (IPQ-R). Psychol Health, 17, 1-16.

NCRP-National Cancer Registry Program (2008). Three-year reports of population based cancer registries 2006-2008. Accessed November 18, 2014. http://www.ncrpindia.org/ Reports/PBCR_2006_2008.aspx

Rao RSP, Suma N, Nair NS, Kamath VG (2005). Acceptability and effectiveness of a breast health awareness programme for rural women in India. Indian J Med Sci, 59, 396-402.

Ray K, Mandal S (2004). Knowledge about cancer in West Bengal-a pilot survey. Asian Pac J Cancer Prev, 5, 205-12.

Raychaudhuri S, Mandal S (2012b). Socio-demographic and behavioural risk factors for cervical cancer and knowledge, attitude and practice in rural and urban areas of North Bengal, India. Asian Pac J Cancer Prev, 13, 1093-6.

Raychaudhuri S, Mandal S (2012a). Current status of knowledge, attitude and practice (KAP) and screening for cervical cancer in countries at different levels of development. Asian Pac J Cancer Prev, 13, 4221-7.

Robb KA, Miles A, Wardle J (2004). Demographic and psychosocial factors associated with perceived risk for colorectal cancer. Cancer Epidemiol Biomarkers Prev, 13, 366-72.

Roy B, Tang TT (2008). Cervical cancer screening in Kolkata, India: beliefs and predictors of cervical cancer screening among women attending a women's health clinic in Kolkata, India. J Cancer Educ, 23, 253-9.

Roychowdhury S, Roychowdhury G, Sen U (2005). Assessment of awareness level on tobacco and smoking habits as risk factors for cancer among lung and laryngeal cancer patients in Kolkata-A case control study. Asian Pac J Cancer Prev, 6, 332-6.

Rutten LF, Hesse BW, Moser RP, McCaul KD, Rothman AJ (2009). Public perceptions of cancer prevention, screening and survival: comparison with state-of-science evidence for colon, skin and lung cancer. J Cancer Educ, 24, 40-8.

Saha A, Chaudhury AN, Bhowmik P, Chatterjee R (2010). Awareness of cervical cancer among female students of premier colleges in Kolkata, India. Asian Pac J Cancer Prev, 11, 1085-90.

Satija A (2012). Cervical cancer in India. Center for chronic disease control. Retrieved on April 5th.

Sheeran P, Harris PR, Epton T (2014). Does heightening risk appraisals change people's intentions and behavior? A metaanalysis of experimental studies. Psychol Bull, 140,511-43.

Shukla R (2010). How India earns, spends and saves: unmasking the real India. New Delhi: Sage Publications India Pvt, Ltd.

Somdatta P, Baridalyne N (2008). Awareness of breast cancer in women of an urban resettlement colony. Indian J Cancer, 45, 149-53.

Takiar R, Nadayil D, Nandakumar A (2010). Projections of number of cancer cases in India (2010-2020) by cancer groups. Asian Pac J Cancer Prev, 11, 1045-9.

Weinman J, Petrie KJ (1997). Illness perceptions: a new paradigm for psychosomatics? J Psychosom Res, 42, 113-6.

Yasmeen J, Qurieshi MA, Manzoor NA, Asiya W, Ahmad SZ (2010). Community-based screening of cervical cancer in a low prevalence area of India: a cross sectional study. Asian Pac J Cancer Prev, 11, 231-4. 